\title{
CORRECTION
}

\section{Author Correction: Neddylation of PTEN regulates its nuclear import and promotes tumor development}

Ping Xie, Zhiqiang Peng, Yujiao Chen, Hongchang Li, Mengge Du, Yawen Tan, Xin Zhang, Zhe Lu, Chun-Ping Cui, Cui Hua Liu (D, Fuchu He and Lingqiang Zhang (iD

Cell Research (2021) 31:374; https://doi.org/10.1038/s41422-021-00470-4

Correction to: Cell Research https://doi.org/10.1038/s41422-02000443-z, published online 09 December 2020

We apologize for three errors that we just found in the paper published online on 9 December 2020. The original Fig. 5j was inconsistent with the raw data, and the correct version is provided below. There is an inaccurate label in the original
Fig. 9k. The label "Grade $(n=18)$ " below the immunohistochemical staining images should be "Grade I $(n=18)$ ". In addition, data in Fig. 9i-k were analyzed by Student's $t$ test. "Data were analyzed using the Kruskal-Wallis test" in the original figure legend should be removed. The correct version is shown below. These corrections do not affect the description and conclusion of this article.

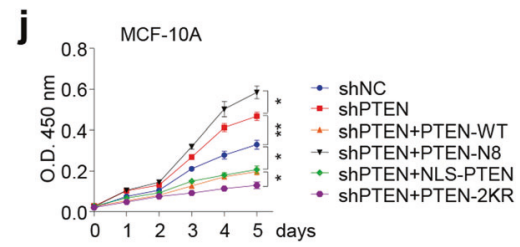

Fig. 5 PTEN neddylation promotes tumorigenesis. j shNC, shPTEN, shPTEN-resistant PTEN-WT, 2KR, NLS-PTEN, PTEN-Nedd8 stable cell lines were generated in MCF-10A cells. CCK8 assay was performed in the indicated cells.
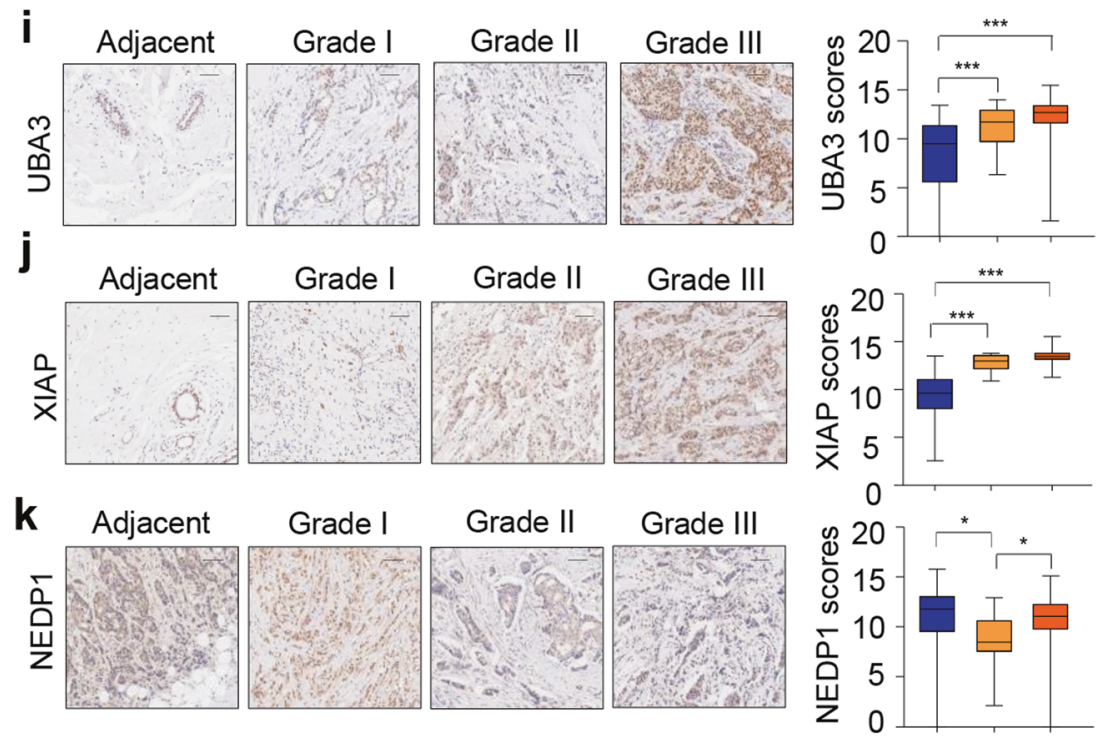

Adjacent ( $\mathrm{n}=99)$

$\square$ Grade I (n=18) $\square$ Grade II/III (n=135)

Fig. 9 Positive correlation of PTEN neddylation with neddylation enzymes in breast cancer. i-k Representative images from immunohistochemical staining of UBA3 (i), XIAP (j) and NEDP1 (k) in different grades are shown. The expression scores are shown as box plots. Scale bars, $100 \mu \mathrm{m}$. ImageJ was used to perform semi-quantitative analysis. 